\title{
A cryogenic imaging X-ray spectrometer for XEUS read out by frequency-division SQUID multiplexers
}

Piet A. J. de Korte, John J. van Baar, Norman H. R. Baars, Frank E. Bakker, Wouter M. Bergmann Tiest, et al.

Piet A. J. de Korte, John J. van Baar, Norman H. R. Baars, Frank E. Bakker, Wouter M. Bergmann Tiest, Marcel P. Bruijn, Alexander Germeau, Henk F. C. Hoevers, Mikko Kiviranta, Eric Krouwer, Jan van der Kuur, Marco P. Lubbers, Wim A. Mels, Marcel L. Ridder, Heikki Seppae, Remco J. Wiegerink, "A cryogenic imaging X-ray spectrometer for XEUS read out by frequencydivision SQUID multiplexers," Proc. SPIE 5501, High-Energy Detectors in Astronomy, (29 September 2004); doi: 10.1117/12.552933

Event: SPIE Astronomical Telescopes + Instrumentation, 2004, Glasgow, United Kingdom 


\title{
A cryogenic imaging X-ray spectrometer for XEUS read out by frequency-division SQUID multiplexers
}

\author{
Piet A.J. de Korte ${ }^{*}$, John J. van Baar ${ }^{2}$, Norman H.R. Baars ${ }^{1}$, Frank E. Bakker ${ }^{1}$, Wouter M. Bergmann \\ Tiest $^{1}$, Marcel P. Bruijn ${ }^{1}$, Alexander Germeau ${ }^{1}$, Henk F.C. Hoevers ${ }^{1}$, Mikko Kiviranta ${ }^{3}$, Eric Krouwer ${ }^{1}$, \\ Jan van der Kuur ${ }^{1}$, Marco P. Lubbers ${ }^{1}$, Wim A. Mels ${ }^{1}$, Marcel L. Ridder ${ }^{1}$, Heikki Seppae ${ }^{3}$, and Remco J. \\ Wiegerink $^{2}$ \\ ${ }^{1}$ SRON National Institute for Space Research, Sorbonnelaan 2, 3584 CA Utrecht, the Netherlands \\ ${ }^{2} \mathrm{MESA}^{+}$Research Institute, University of Twente, P.O. Box 217, 7500 AE Enschede, the Netherlands \\ ${ }^{3}$ VTT Microsensing, Tietotie 3, 02150 Espoo, Finland
}

\begin{abstract}
A micro-calorimeter array with superconducting transition-edge sensors read out by a SQUID-based frequency-domain multiplexer is under development for the X-ray imaging spectrometer on board ESA's X-ray Evolving Universe Spectroscopy (XEUS) mission. The XEUS requirements are 2 and $5 \mathrm{eV} \mathrm{FWHM} \mathrm{energy} \mathrm{resolution} \mathrm{for} 2$ and $7 \mathrm{keV} \mathrm{X-rays,}$ respectively. An array of $32 \times 32$ pixels with 250 micron square pixels is envisaged.

SRON and $\mathrm{MESA}^{+}$have developed 5 x 5 imaging micro-calorimeter prototype arrays along a bulk micromachining and a surface micromachining route. The present state of array design and development with emphasis on pixel-to-pixel performance measurements of thermal and I-V characteristics, sensor noise and energy resolution are presented.

SRON and VTT are developing frequency-domain multiplexing with SQUID current amplifiers to read out the $32 \mathrm{x}$ 32 array. The concept for the frequency division multiplexing read-out will be presented and its performance characteristics discussed. Recent results of sensor operation under AC-bias $(500 \mathrm{kHz})$ are presented.
\end{abstract}

Keywords: Cryogenic micro-calorimeters, transition-edge sensors (TES), SQUIDs, imaging X-ray spectrometer, X-ray astronomy, XEUS, frequency-domain multiplexing (FDM)

\section{INTRODUCTION}

Cryogenic imaging spectrometers most likely will have huge impact on the future of X-ray astronomy, since they combine high detection efficiency over a wide spectral band with high spectral resolution, adequate imaging and fast thermal response time. About 20 years after the initial presentation of a single pixel X-ray micro-calorimeter by Moseley, Mather and McCammon $^{1}$ in 1984, the first 36-pixel micro-calorimeter array based on doped-Si thermistors ${ }^{2}$ will be launched on the Japanese Astro-E2 mission in early 2005. Since the initial conception of X-ray micro-calorimeters, several new types have been proposed with the Transition Edge Sensor ${ }^{3}$ (TES) being one of the most promising today. TES employ superconducting-to-normal phase transition thermometers that can be operated in extreme electro-thermal feedback (ETF) through the use of voltage bias. ETF results in stable responsivity, fast (50-100 $\mu$ s) response time, and $2-4 \mathrm{eV}$ energy resolution.

An advantage of TES-based devices over the ones developed for Astro-E2, is that their fabrication is compatible with thin film deposition, photo-lithography, and micromachining techniques, thereby potentially enabling the production of very large pixel arrays. For the next generation X-ray missions, like NASA's Constellation-X and ESA's X-ray Evolving Universe Spectroscopy mission XEUS, 32 x 32 pixel arrays are foreseen. TES-based micro-calorimeters can benefit from their low output impedance, typically $1-10 \mathrm{~m} \Omega$, since that matches extremely well with the input impedance of SQUID current amplifiers. Furthermore the typical noise temperature of a SQUID, typically $<100 \mu \mathrm{K} @ 1 \mathrm{MHz}$, is substantially

*p.a.j.de.korte@sron.nl; phone +31 30 2535710; fax +31 30 2540860; http://www.sron.nl 
smaller than that of the TES, which equals approximately $100 \mathrm{mK}$, leaving ample design margin. Moreover, the SQUID bandwidth of at least $100 \mathrm{MHz}$ leaves ample space to multiplex several signal chains into one amplifier chain.

\section{TES PRINCIPLE, DESIGN AND SINGLE PIXEL PERFORMANCE}

The basic physics and theoretical performance of a voltage-biased detector with a superconducting-to-normal phase transition thermometer are well established ${ }^{3,4}$. A micro-calorimeter consists of an absorber and thermometer with total heat capacity $C$, weakly coupled to a heat bath via a thermal conductance $G$. The TES thermometer is characterized at its operating point by temperature $T$, resistance $R$, and temperature coefficient of resistance $\alpha=\mathrm{d}(\log R) / \mathrm{d}(\log T)$. Voltage bias in combination with a positive temperature coefficient of resistance $\alpha$ introduces negative electro-thermal feedback, thereby stabilizing and linearising the device response and shortening the response time.

In the limit of strong ETF, $T>>T_{\text {bath }}$, the energy resolution for small signals can be written as:

$\Delta E_{F W H M}^{\text {th }}=4 \sqrt{\ln 4}[\gamma . n]^{1 / 4} \sqrt{k_{B} T^{2} \frac{C}{\alpha}}$

with $n \approx 3-4$ the power law exponent of the temperature dependent heat flow to the bath, and $\gamma \approx 0.5$ a factor taking into account the temperature gradient over the heat link. If one approximates the thermometer transition by an exponential relation, $R(T)=R_{N} \cdot \exp \left(\left(T-T_{C}\right) / \delta T\right)$ with $R_{N}$ the normal state resistance, $T_{C}$ the transition temperature and $\delta T$ the transition width, the energy at which the thermometer just saturates can also be written as a function of $C / \alpha$, i.e.:

$E_{S A T}=-\ln [r] \frac{C}{\alpha} T$, with $r=R / R_{N}$

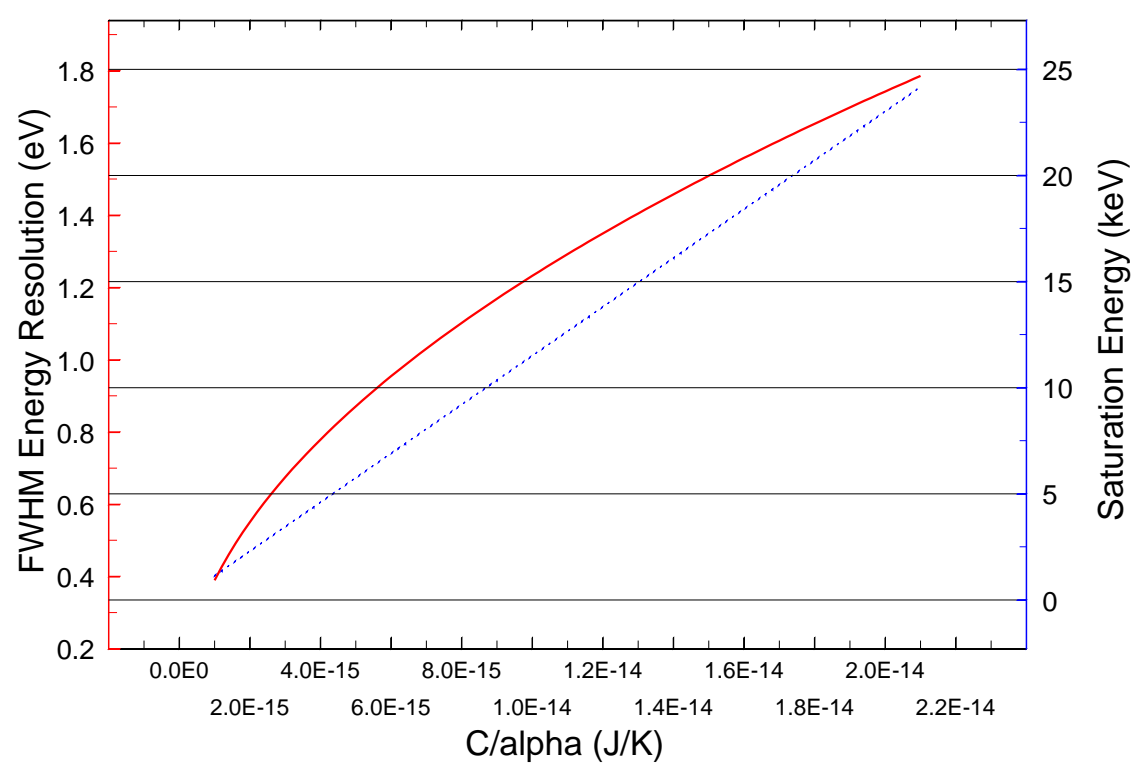

Figure 1: Energy resolution (solid line) and saturation energy (dotted line) as a function of $\mathrm{C} / \alpha$ for $\mathrm{T}=100 \mathrm{mK}$ and $\mathrm{r}=0.1$

SRON indicate a degradation of the energy resolution for $5.9 \mathrm{keV}$ X-rays by approximately a factor 1.5 compared to the small signal resolution.
So both design parameters are a function of $C / \alpha$ as shown in fig. $1 \mathrm{In}$ case one requires a saturation energy of $10 \mathrm{keV}$, which is the case for Constellation-X and XEUS, one should design for $C / \alpha$ equal to about 9 $10^{-15}$. In that case the limiting energy resolution is about $1.2 \mathrm{eV}$.

So far the best resolution obtained for TES devices, crudely optimized for the $10 \mathrm{keV}$ energy range, equals $2.4 \mathrm{eV}$ and $3.7 \mathrm{eV}$ for 1.5 and

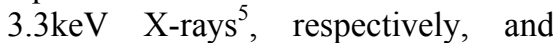
$3.9 \mathrm{eV}$ for $5.9 \mathrm{keV} \mathrm{X}$-rays $^{6}$ (see fig. 2). These resolutions are about a factor 23 worse than the theoretical limit. By now the origin of this discrepancy is understood to some extent. One of the factors originates from the fact that the response of the sensor becomes nonlinear for large signals, while the algorithm (Wiener filter) presently used for the energy determination of $\mathrm{X}$-ray events is optimized for a linear response. Simulations carried out for single pixel devices manufactured at 


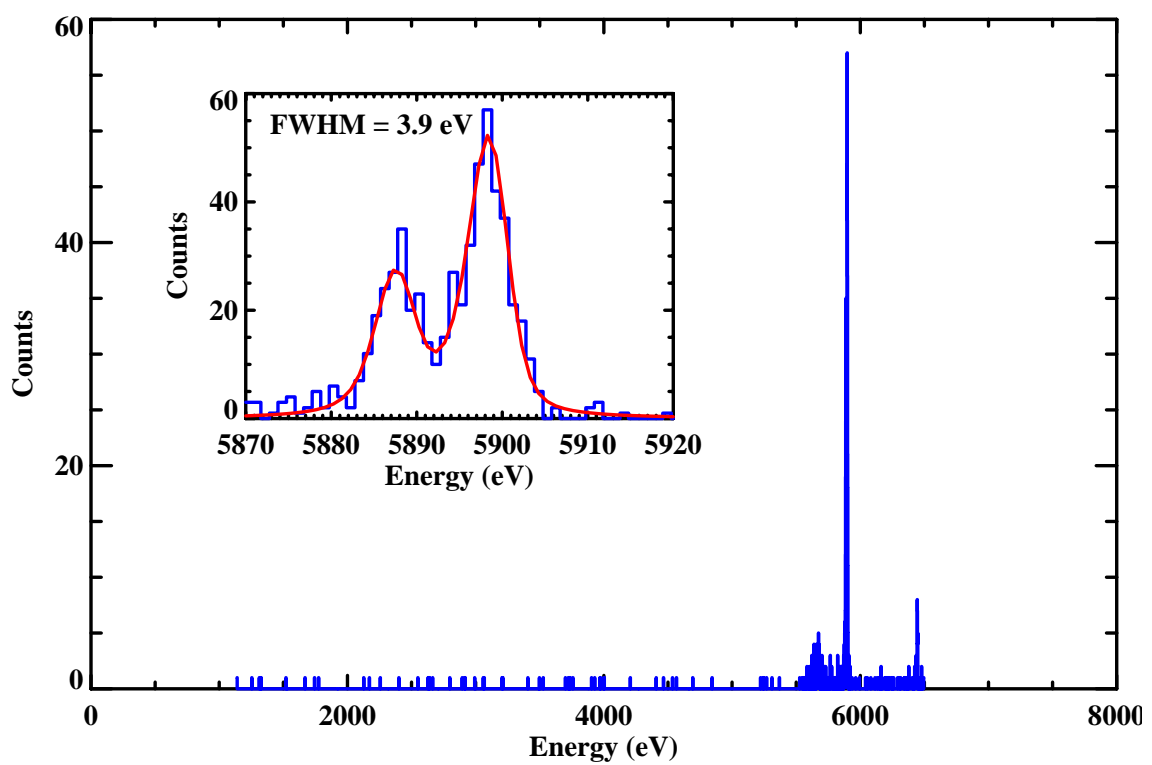

Figure 2: The best energy resolution measured for $5.89 \mathrm{keV}$ X-rays so far equals 3.9 eV FWHM in combination with $100 \mu$ s response time

The second factor degrading the energy resolution is related to the presence of internal fluctuation noise in TES devices originating from internal temperature fluctuations between the resistive TES and the absorber heat capacitance ${ }^{7,8}$. This internal fluctuation noise degrades the energy resolution of TESs, as used by SRON (TiAu bilayer with $R_{N} \approx 0.3 \Omega / \square$ ), with a factor $1.5-2$, with respect to the theoretical resolution from eq. 1 .

In case of low-ohmic TES devices, like the $R_{N} \approx 0.01 \Omega / \square \mathrm{MoAu}$ TES used by GSFC and NIST, the internal fluctuation noise doesn't play a role and the origin of the excess noise in those devices is not understood yet. The excess noise as observed by GSFC and NIST also leads to a resolution degradation of about a factor 1.5 - 2 .

For the large signal energy resolution degradation a potential solution has been offered by Fixen et al. ${ }^{9}$ through the development of a pulse estimation algorithm for non-linear detectors with non-stationary noise. For the suppression of excess noise TESs equipped with zebra type stripes ${ }^{8}$ have been proposed. They suppress the excess noise level but at the expense of degradation of the temperature coefficient of resistance $\alpha$, so that it might be hard to effectively use it to improve the energy resolution.

The final relation, important for the sensor design, is the effective response speed that can also be expressed in terms of $C / \alpha$ :

$$
\tau_{e}=\frac{C}{G} \frac{1}{1+L_{0}} \approx \frac{C n}{G \alpha} \approx \frac{C}{\alpha} \frac{T}{P}
$$

with $\mathrm{L}_{0}$ the electro-thermal loop gain and $\mathrm{P}$ the equilibrium bias power flow to the heat bath. For $C / \alpha \approx 9.10^{-15}$ and $T=100$ $\mathrm{mK}$ a bias power of about $10 \mathrm{pW}$ is required for $\tau_{\mathrm{e}} \approx 100 \mu \mathrm{s}$. The response time measured for TES devices ranges from $50 \mu \mathrm{s}$ to several ms. It will be difficult to make them much faster than $50 \mu$ s, given the device speed limits itself ${ }^{4}$, the bias power limits for an imaging array, and the increasing demand on the electronics slew rate.

\section{ARRAY DEVELOPMENT AND PERFORMANCE}

Both Constellation-X and XEUS require an imaging array of at least 32 x 32 pixels. The first step has been to design and develop a single pixel layout that is consistent with use in an array. A schematic of such a pixel is shown in fig. 3. The mushroom shaped absorber allows for close packing, since it makes space available for the connecting wiring. The thermal conductance to the heat bath can be tuned by means of structuring the $\mathrm{Si}_{\mathrm{x}} \mathrm{N}_{\mathrm{y}}$ membrane.

Taking this type of pixel layout as design standard, SRON and MESA ${ }^{+}$are developing two process routes for the fabrication of $5 \times 5$ pixel proto-type imaging arrays ${ }^{10,11}$. The difference is in the formation of the supporting structure. In the bulk micromachining route, shown in fig. 4 , the structure is formed by etching deep, vertical slots in the backside of a Si (110) wafer by the use of anisotropic wet etching. The resulting walls have a (111) orientation and a very smooth surface. The advantage of the bulk micromachining route is its relative simplicity. After etching the beams, the pixel fabrication is performed on the free-standing membranes. Disadvantage of this route is its vulnerability to mechanical stress, which might 


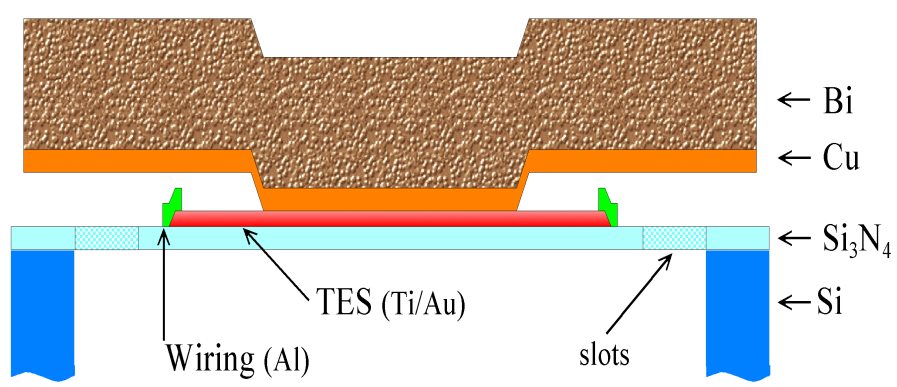

Figure 3: Conceptual design of an array pixel. The overhanging absorber allows for close packing, since it creates space for slots in the $\mathrm{Si}_{\mathrm{x}} \mathrm{N}_{\mathrm{y}}$ membrane to tune the heat conductance, and space for the connecting wiring. The aspect ratio of this and the two following figures is distorted. The pixel size is about $250 \mu \mathrm{m}$ and the absorber thickness about $7 \mu \mathrm{m}$.

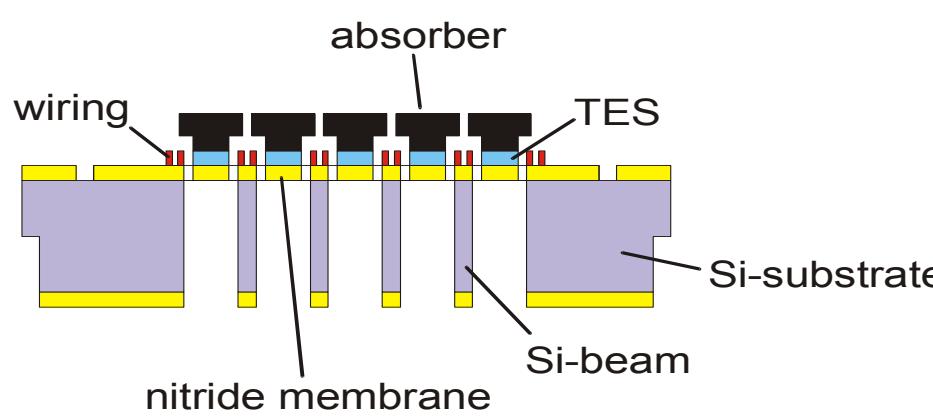

Figure 4: Schematic side view of a pixel array, formed by bulkmicromachining. Slots are wet-etched into a $\mathrm{Si}_{\mathrm{x}} \mathrm{N}_{\mathrm{y}}$ coated $\mathrm{Si}$ (100) wafer. The pixels structure on the top is formed by e-beam evaporation, sputter deposition, etching and lift-off processes after the definition of the beams.

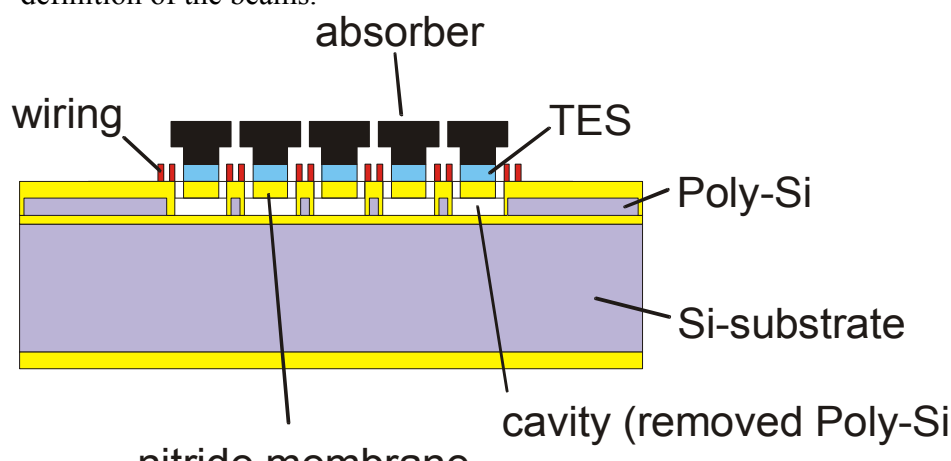

nitride membrane

Figure 5: Schematic side view of a pixel array formed by surface micromachining. A poly-Si sacrificial layer is used to create a cavity under each pixel. Access to the cavity is at present dry-etched from the top. The last step in the process is the removal of the poly-Si by means of TMAH. easily destroy a whole column of pixels. Another drawback is the finite thermal conductance of the silicon beams that tends to limit the array size. It was hoped for that the smoothness of the (111) beam walls would result in a very long phonon mean free path and thereby in good heat conduction. Measurements indicate that the phonon $\mathrm{mfp}$ is $110 \mu \mathrm{m}$ for a $40 \mu \mathrm{m}$ wide Si-beam. For a 32 × 32 pixel array and a bias power of $10 \mathrm{pW} / \mathrm{pixel}$ this would result in a temperature of more than $200 \mathrm{mK}$ for the Si-beams and the Si-chip. It has been shown at SRON that the cooling capacity can be significantly improved by coating the Sibeams and the bottom of the detector chip with a $\mathrm{Cu}$ layer of typically $0.7 \mu \mathrm{m}$ thick. Measurements in combination with simulations show that a $\mathrm{Cu}$ coated 32 x 32 pixel array $(10 \mathrm{pW} /$ pixel $)$ will reach a temperature of $47 \mathrm{mK}$ at the center of the beams and $40 \mathrm{mK}$ at the chip, when coupled to a heat bath at $20 \mathrm{mK}$. These temperatures are quite acceptable.

The other process, see fig. 5, is based on surface micromachining. In this process a shallow cavity underneath the membrane is created by surface micromachining techniques using a poly-Si sacrificial layer. The poly-Si in the cavity is removed at the end of the process by wet TMAH etching from the front side. The advantage of this route is that the structure is mechanically more rigid and that it has better thermal conductance and therefore lower thermal cross-talk. This structure opens the opportunity to bury wiring at the bottom of the cavity, so that substantially larger arrays will be possible than for the bulk micromachining route. Membranes tend to stick at the bottom of the cavity, which has been solved by manufacturing small bumps on the inside of the membrane. A potential problem arises from the removal of the poly-Si in the last step in the process, which takes about $5-8$ hours at $85^{\circ} \mathrm{C}$. It has been found that both the $\mathrm{Cu}$-layer of the absorber and the $\mathrm{Au}$ layer of the TES are chemically etched by TMAH. Tests with different protection layers have shown that a $5 \mathrm{~nm}$ Ti layer provides excellent protection of both the $\mathrm{Cu}$ and the Au layers. Another complication is that the transition temperature of the TES decreases during TMAH etching with approximately $20 \mathrm{mK}$, almost certainly a result of enhanced inter diffusion of $\mathrm{Au}$ and Ti due to the extended period at $85^{\circ} \mathrm{C}$. This shift can be taken into account in the sensor design by starting with a TiAuTi TES with a transition temperature $20 \mathrm{mK}$ higher than finally aimed for. Another way out might be the use of thermally more stable bi-layers like $\mathrm{MoAu}$ and $\mathrm{MoCu}$. 
Proto-type 5 x 5 pixel micro-calorimeter arrays have been produced and tested for both manufacturing routes. In each of the arrays three pixel (side, center, corner) are connected and fully operational. The other pixels are interconnected and equipped with heaters enabling uniform temperature operation of the whole array. A micrograph of both array type is shown in fig. 6 and 7.

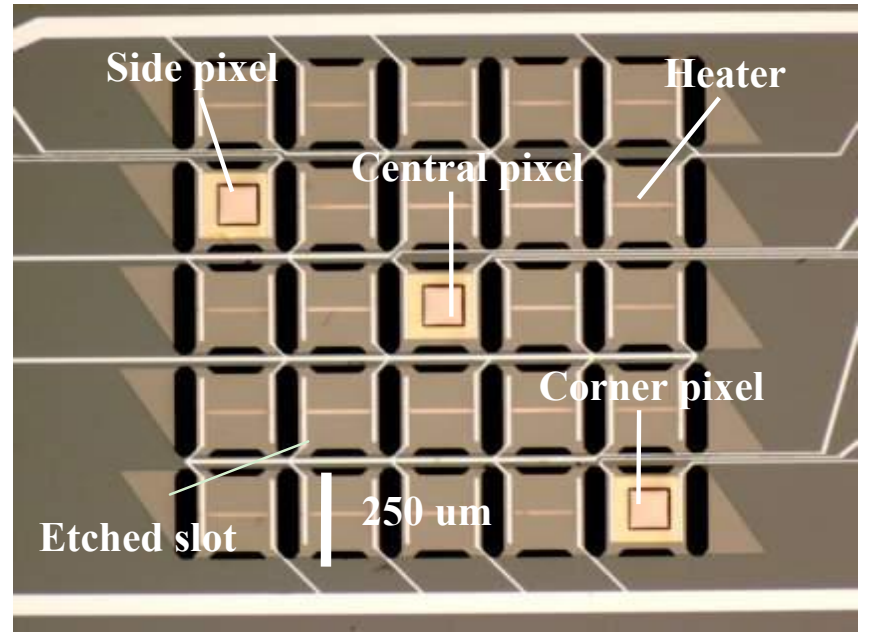

Figure 6: 5 x 5 pixel prototype array fabricated by bulkmicromaching.

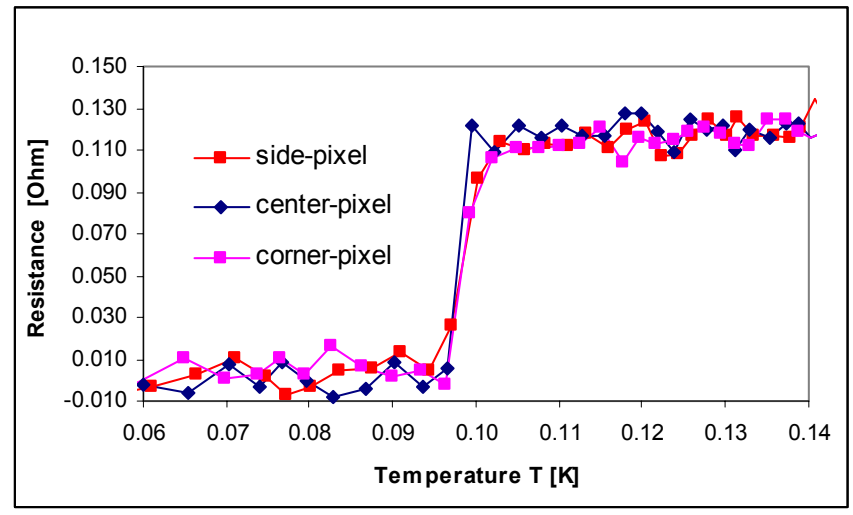

Figure 8: Measured $\mathrm{R}(\mathrm{T})$ curves for the three pixels of the bulk micro-machined array shown in fig. 6

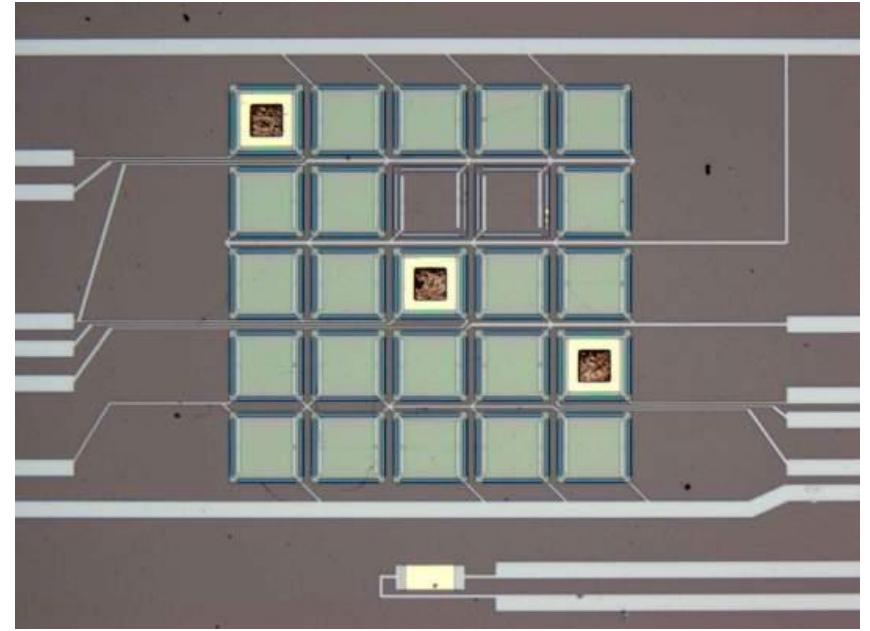

Figure 7: 5 x 5 pixel prototype array fabricated by surface micromachining

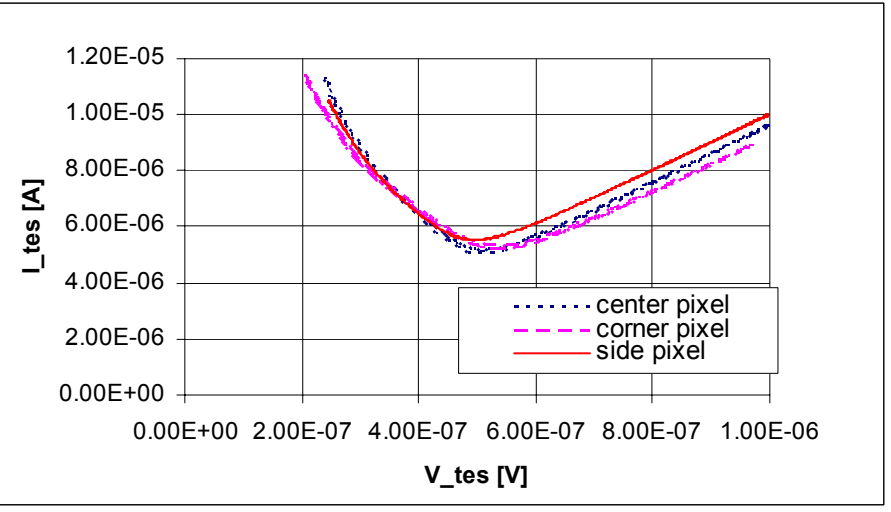

Figure 9: Measured I -V curves for the three pixels of the bulk micromachined array shown in fig. 6

For both array type the bias power was found to be $2-4 \mathrm{pW} /$ pixel whereas they were designed for $10 \mathrm{pW}$. After extensive analysis and experiments it was concluded that the thermal conductance of the $\mathrm{Si}_{\mathrm{x}} \mathrm{N}_{\mathrm{y}}$ grown on $\mathrm{Si}$ (110) and poly-Si was substantially lower than the thermal conductance of previously used $\mathrm{Si}_{\mathrm{x}} \mathrm{N}_{\mathrm{y}}$ on $\mathrm{Si}(100)$ wafers. In case the thermal coefficient of conductance is expressed as $\kappa(T)=\kappa_{0} \cdot T^{n-1}$ our data are described with $n=3.6$ and with a $\kappa_{0}$ that for $\mathrm{Si}_{\mathrm{x}} \mathrm{N}_{\mathrm{y}}$ grown on $\mathrm{Si}$ (110) or poly-Si is equal to $9 \pm 1 \mathrm{~mW} / \mathrm{K}^{\mathrm{n}} / \mathrm{m}$ and for $\mathrm{Si}_{\mathrm{x}} \mathrm{N}_{\mathrm{y}}$ grown on $\mathrm{Si}(100) 39 \pm 9 \mathrm{~mW} / \mathrm{K}^{\mathrm{n}} / \mathrm{m}$. Although more research is going on to understand this unexplained difference, SRON has started a redesigning its pixels in order to meet the $100 \mu \mathrm{s}$ response time by increasing the heat flow to $10 \mathrm{pW} /$ pixel. 
$\mathrm{R}(\mathrm{T})$ and I - V curves have been measured for each of the three pixels in the bulk micro-machined array shown if fig. 6 . The $\mathrm{R}(\mathrm{T})$ curves are shown in fig. 8. As can be observed from these measurements the uniformity over this array is quite good. The I-V curves of the three pixels are subsequently shown in fig. 9. Also these curves turn out to be quite similar. Analysis of these I - V curves confirms the too low power plateau, which is actually $2.2 \mathrm{pW} /$ pixel. The I - V curves also show a good temperature coefficient of resistance $\alpha$ of about $70-100$ under bias conditions. For the surface micro-machining route the $\mathrm{R}(\mathrm{T})$ measurements turn out to be quite similar to those shown in fig. 8. X-ray energy resolution measurements were performed on several pixels of the bulk micro-machined arrays. The response time of the pixels varies from 0.2 to $3 \mathrm{~ms}$ for membrane legs with a width ranging from 200 to $15 \mu \mathrm{m}$, respectively. The best energy resolution measured for a few pixels equals $6 \mathrm{eV}$ FWHM with excursions up to $30 \mathrm{eV}$ FWHM. It is not clear so far why the measured resolution is worse than the $4-5 \mathrm{eV}$ FWHM resolution measured quite reproducible on single pixels. It most probably finds its origin in deteriorated experimental conditions. Work has started to improve the EM-shielding in our experimental set-up and we are in the process to move to fully differential cabling and electronics.

\section{SINGLE PIXEL READ-OUT AND MULTIPLEXER DEVELOPMENT}

The output impedance of a TES is approximately equal to its bias point resistance $R$, which in case of SRON's high-ohmic TiAu thermometers, equals $\approx 10 \mathrm{~m} \Omega$ for $r=0.1$. This is less than expected on the basis of the TiAu sheet resistance, due to the central copper stem of the absorber (fig. 3). The noise temperature of the TES equals $\gamma \cdot n . T \approx 2 T$ with $T$ the temperature at the bias point, typically $100 \mathrm{mK}$ for most TES devices. A SQUID current amplifier is the obvious choice as it has a typical noise temperature of $100 \mu \mathrm{K}$ at $1 \mathrm{MHz}$ and an input impedance well-suited to read out low ohmic devices. The mutual inductance of the SQUID input coil is designed for proper noise matching. In our case an input current noise, $i_{n}=10$ $\mathrm{pA} / \mathrm{Hz}^{1 / 2}$, is required.

More problematic is the relatively small SQUID dynamic range $D R$. Based on the sensor characteristics its requirement is defined by:

$D R \equiv \frac{\Delta I}{i_{n}}=\beta \frac{(1-r) E_{s a t}}{\Delta E_{r m s} \sqrt{\tau_{e f f}}} \quad\left[\mathrm{~Hz}^{1 / 2}\right]$

where $\beta=3$ takes care of the fact that the electronics should not degrade the sensor performance. For the XEUS mission we use $E_{\text {sat }}=10 \mathrm{keV}, D E_{r m s}=0.9 \mathrm{eV}$, and $\tau_{\text {eff }}=50 \mu \mathrm{s}$, so that the required dynamic range equals $\approx 410^{6} \mathrm{~Hz}^{1 / 2}$. The SQUID dynamic range equals about $0.25 \Phi_{0} / \Phi_{\mathrm{n}}$, which, for good SQUIDs, ranges from about $510^{5}-2.510^{6} \mathrm{~Hz}^{1 / 2}$, which is smaller than actually required. In order to increase the dynamic range of the SQUID current amplifier use of feedback by means of a so-called flux-locked-loop (FLL) is the most common approach.

The bandwidth of the signal is limited by its rise time $\tau_{\mathrm{R}}$ that can be tuned by the amount of inductance in the bias circuit. However, $\tau_{\mathrm{R}}$ has to comply with the criteria for electro thermal stability ${ }^{12}$. In case of optimum damping $\tau_{\text {eff }}=\tau_{\mathrm{R}}(3+$ $\left.5^{1 / 2}\right) / 2$ at the bias point resistance $R$. In case of a saturated signal $\tau_{\mathrm{R}}$ becomes a factor $r=0.1$ faster, i.e. $1.9 \mu$ s. For XEUS this sets the minimum bandwidth requirement at $84 \mathrm{kHz}$.

In case of an $N$ x $M$ pixel array the read out of each pixel by its own SQUID becomes quickly prohibitive. The designed cooling power for the XEUS ADR equals approximately $5.5 \mu \mathrm{Wh} @ 35 \mathrm{mK}$, so that the heat input through the wiring, at least 4 twisted wire-pairs for each SQUID/TES combination, and that of the SQUIDs $(\approx 1 \mathrm{nW} / \mathrm{SQUID})$ will result in uninterrupted observation times of at best $\leq 4$ hours. So read out schemes that reduce the amount of wiring and the number of powered-on SQUIDs will be required to increase uninterrupted observation periods to at least 24 hours. Two type of multiplexing schemes, time-domain-multiplexing (TDM) ${ }^{12,13}$ and frequency-domain-multiplexing (FDM) ${ }^{14,15}$ are under development for this purpose. TDM employs SQUIDs as switches to connect multiple TESs to a single read-out SQUID alternatingly, so that the signals from the various pixels become separated in time. FDM exploits the TES as a modulating element, by applying an AC bias voltage instead of the commonly applied DC bias. With the pixels biased at individual bias frequencies, the signals become separated in frequency space and can be amplified by a single SQUID amplifier. A schematic diagram of such a FDM system is shown in fig. 10. 


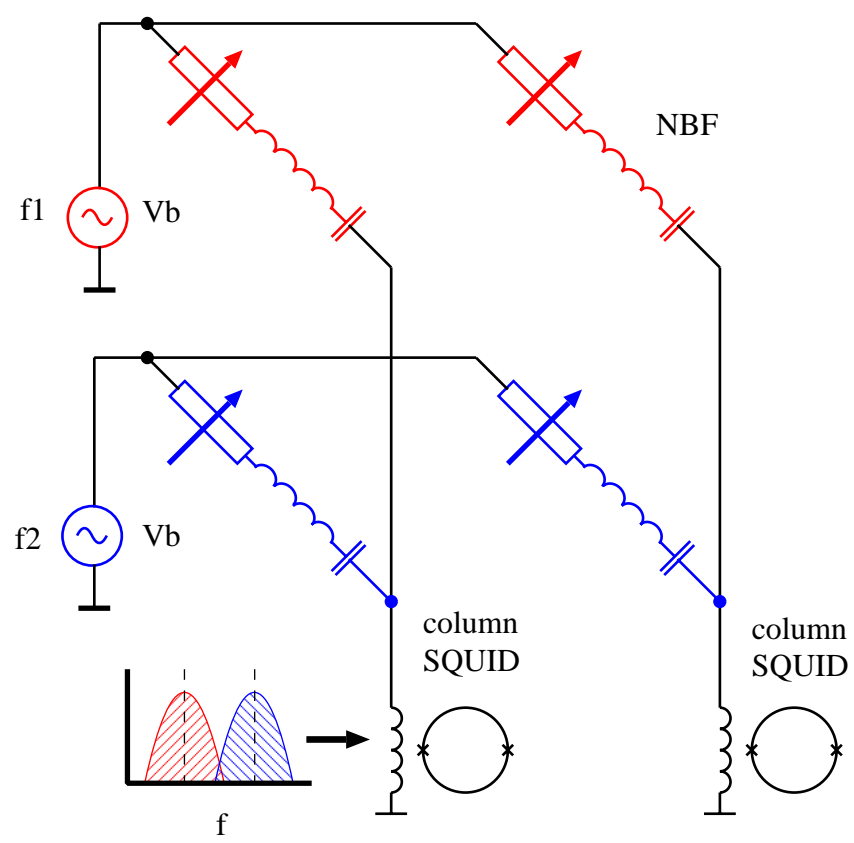

Figure 10: Schematic FDM layout with current summing topology
Both multiplexing methods are in principle equivalent, and a discussion of some of the trade-offs is found in literature ${ }^{16}$. What is not discussed in literature sofar is that the specific requirements for XEUS make FDM the optimum choice. This can be most easily seen by comparison of the dynamic range requirement. In case of TDM the required dynamic range increases with $M^{1 / 2}$, where $M$ is the number of multiplexed pixels, as aliasing of broadband SQUID noise would otherwise spoil the energy resolution of the pixels. For FDM with bias-current cancellation, the dynamic range is a function of the number and amplitude of coincident signals in the $M$ multiplexed pixels However, the XEUS requirements are such that coincident absorption of two high energy photons in $M$ pixels is extremely unlikely, actually most pixels in an X-ray imager are essentially empty on the time scale of a single event. Simulations of typical XEUS observations show that the dynamic range for $F D M$ is essentially independent of $M$, at least up to $M<64$ and count rates up to $5000 \mathrm{c} / \mathrm{s}$ per detector column.

Various summing topologies have been proposed for an FDM read-out system. The first topology proposed is voltage summing into a summing loop ${ }^{17}$ by means of $M$ input transformers to that summing loop. A clear disadvantage of this structure is that only part of the signal energy is

transferred to the SQUID, so that the required energy resolution of the SQUID becomes proportional to $1 / M$. The current summing into a single wire topology, proposed by VTT/SRON ${ }^{14}$, 15 (see fig. 10), feeds the total signal energy into the SQUID, making the SQUID input noise and energy resolution independent of the number of pixels $M$.

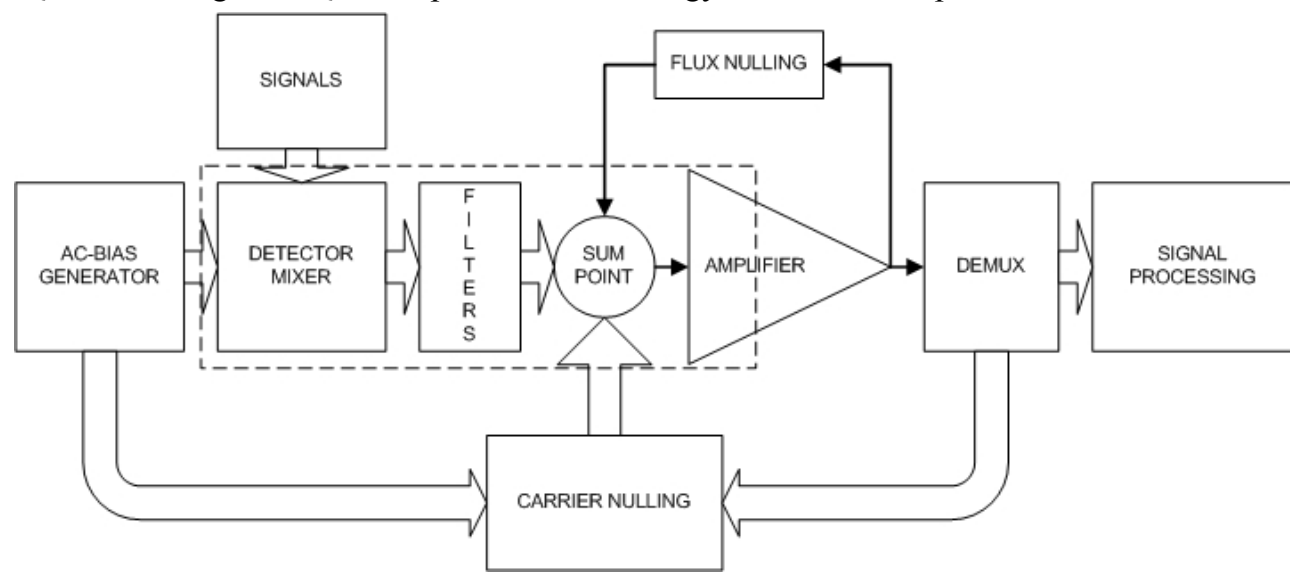

Fig. 11: Schematic of the FDM design with a standard type of flux-locked-loop (FLL). Carrier nulling, reducing the required dynamic range, is implemented as a slow separate feedback loop with high gain. The part within the dashed box is at approximately $100 \mathrm{mK}$
The flux summing topology ${ }^{18}$ is quite analogous to the current summing one. Disadvantage is the potential complexity of the coupling transformer, coupling losses, and capacitive coupling between coils and washer. The use of a multi-input SQUID might solve some of these issues at the cost of SQUID complexity; SQUID flux noise, and cross-talk.

SRON has chosen for the current summing topology shown in fig. 10. In this topology cross-talk is created by the voltage drop over the common impedance that mainly consists of the input inductance $L_{i n}$ of the SQUID. Obviously this kind of cross-talk can be reduced by use of a tuning capacitor in series with the input inductance $L_{i n}$ and by feedback of the SQUID output to the summing node (fig. 11). 
In that case the crosstalk level can be made negligible, i.e. $\delta E / E<10^{-4}$, as long as the SQUID input inductance can be kept below a few $\mathrm{nH}$. Another cross-talk mechanism is the generation of mixing products by coincident signals on the a-linear

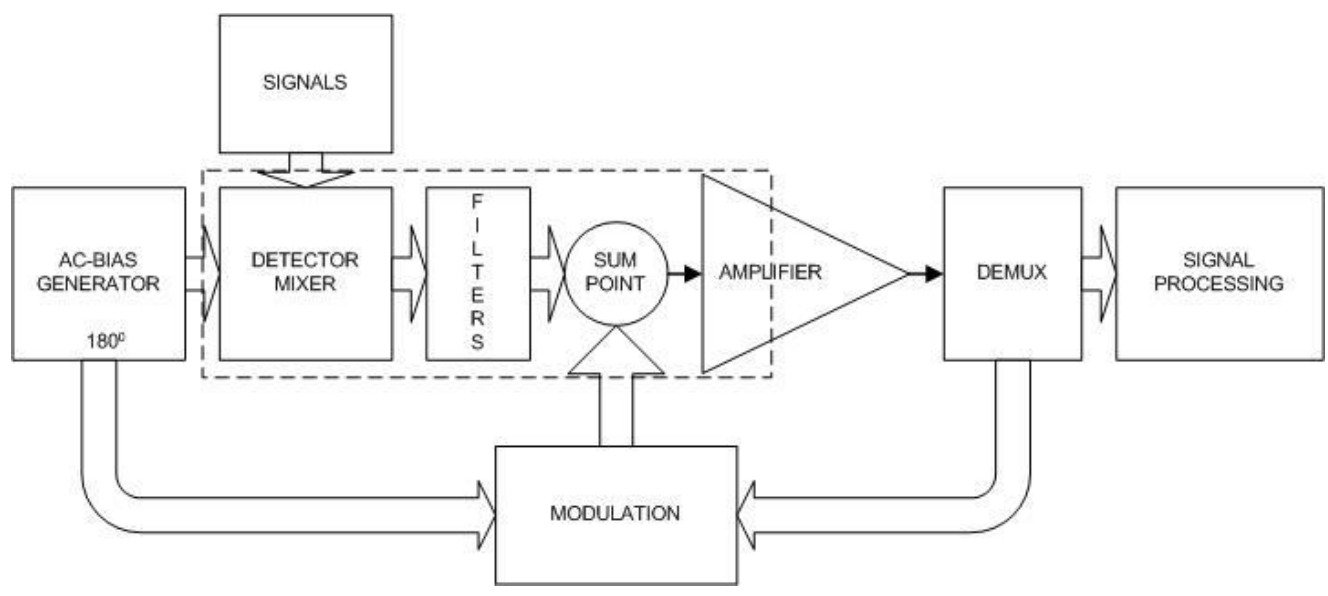

Fig. 12: Schematic of an FDM design making use of base-band feedback for the flux-locked-loop (FLL). Given the high FLL-gain possible in this design a separate feedback loop for carrier nulling is not required. The part within the dashed box is at approximately $100 \mathrm{mK}$.
SQUID response. Taking into account the linearity and noise figures of the SQUID developed by VTT for this project, a FLL feedback gain of about 10 will be required to suppress cross-talk levels due to mixing below the $\delta E / E<$ $10^{-3}$ level for saturated signals. Analysis has shown that this is quite acceptable for the XEUS application. This requirement on the FLL-gain does however limit the available bandwidth of the standard FLL-design shown in fig. 11 to approximately 3 $\mathrm{MHz}$, given the warm amplifier bandwidth and the cable length between warm electronics and SQUID. More FLL-gain and bandwidth becomes available by the use base-band feedback (see fig. 12), making use of the fact that the amplitude and phase of the carriers are known. In that case FLL gains > 200 can be obtained for the information frequency band (100 - $200 \mathrm{kHz})$ so that mixing cross-talk becomes negligible. In this design the carrier frequencies are not limited by FLL-gain requirements and values up to $10 \mathrm{MHz}$ are quite realistic. The frequency limit will now be set by capacitive cross-talk between cables, equivalent series resistance in the filter capacitor, and back-action noise from the SQUID.

Most of the performance results of TES-based micro-calorimeters have been demonstrated using a DC-bias source. For FDM, however, AC bias is required since the TES is employed as a modulator. Therefore, experimental demonstration of good performance under AC bias is essential for the applicability of FDM. Two experiments showing similar X-ray energy resolution under $\mathrm{AC}$ and $\mathrm{DC}$ bias have been reported in literature ${ }^{19,20}$ for about 50 and $150 \mathrm{kHz}$. Recently, SRON obtained an energy resolution of $8.8 \mathrm{eV}$ for $5.9 \mathrm{keV}$ X-rays at a modulation frequency of $500 \mathrm{kHz}$, while for DC bias $6 \mathrm{eV}$ has been obtained. The decrease of energy resolution under AC bias at this frequency has been limited by SQUID noise and new experiments with SQUIDs especially developed for this application are underway. SRON recently verified earlier reports of problems with AC biasing low in the $R-T$ transition $^{21,22}$. It has been demonstrated that for sufficiently low series resistance in the bias circuit, the TES can be AC biased over the full range of the $R(T)$ curve, and it has been measured that the $I-V$ curves under $\mathrm{AC}$ and $\mathrm{DC}$ bias are essential equal. A slight remaining difference can be explained by the generation of higher order harmonics under AC bias.

FDM requires an LC blocking filter for each pixel. In order to meet electro thermal stability the inductance $L$ of the filter has to be equal to or smaller than $105 \mathrm{nH}$ for the case of optimum damping. Such coils made on a washer will have a size of approximately $0.3 \times 0.3 \mathrm{~mm}^{2}$. The smallest possible filter capacitance $C, f_{c}=1 / 2 \pi(L C)^{-1 / 2}$, ranges from $240-2.4 \mathrm{nF}$ for a 1 $10 \mathrm{MHz}$ carrier frequency $f_{c}$, respectively. TES operation requires a voltage source with a series resistance smaller than the TES bias resistance of $10 \mathrm{~m} \Omega$. Resistive losses in the band pass filters will be dominated by losses in the capacitor dielectrics as long as superconducting materials are used for the inductance coils and the capacitor plates. These losses can be expressed as an equivalent series resistance $R_{\mathrm{ESR}}$. For $R_{\mathrm{ESR}} \approx 1 \mathrm{~m} \Omega$ the $Q$-factor $\left(1 / Q=\omega C R_{\mathrm{ESR}}\right)$ of the capacitors has to be between 670 and 6700 for carrier frequencies between 1 and $10 \mathrm{MHz}$, respectively. In order to keep the size of the capacitors small dielectrics with a high dielectric constant are preferred. However, experiments on capacitors with $\mathrm{Ta}_{2} \mathrm{O}_{3}\left(\varepsilon_{\mathrm{r}}=17\right)$ and $\mathrm{Nb}_{2} \mathrm{O}_{5}$ 
$\left(\varepsilon_{\mathrm{r}}=43\right)$ dielectrics have shown Q-factors of 71 and $333^{23}$, respectively. Recently, measurements of capacitors with a $\mathrm{Si}_{\mathrm{x}} \mathrm{N}_{\mathrm{y}}$ $\left(\varepsilon_{\mathrm{r}}=7.8\right)$ dielectric showed a Q-factor of 2800 , which is sufficient for this application. The specific capacitance of these capacitors will be in the $2-5 \mathrm{nF} / \mathrm{mm}^{2}$ range. Obvious disadvantage of these low dielectric constant capacitors is their size. In the worst case they range from $120-1.2 \mathrm{~mm}^{2}$ for 1 and $10 \mathrm{MHz}$, respectively. In case the frequency channels are separated by the signal bandwidth of $85 \mathrm{kHz}$, the required frequency range for 32 channels is about $2.7 \mathrm{MHz}$. For the standard FLL design (fig. 11) this band should be placed at the lowest possible frequency, since that allows for maximum gain of the FLL. However, at too low frequency the size of the capacitors will become prohibitive. Tentatively we have chosen for the $1-3$ $\mathrm{MHz}$ band with slightly closer frequency packing. This still allows for a FLL gain of approximately 10, required for dynamic range and SQUID linearization, at the highest frequency and the total capacitor area for a $32 \times 32$ array is estimated at 200 $400 \mathrm{~cm}^{2}$ quite large, but just workable. For the base-band FLL design higher frequencies and larger frequency separations are possible thereby reducing the required area for the filter capacitors.

The accuracy of the filter components will be dominated by the non-uniformity in the dielectric thickness, which is estimated to be of the order of $1 \%$. This may come out to be the limiting factor for the frequency spacing between the carrier frequencies, unless post-production capacitor tuning is possible.

Optimized SQUID design for XEUS is being performed by VTT-Microsensing. Recent results concerning design considerations and performance have been published elsewhere ${ }^{24}$. The key design parameter for SQUIDs for this application is a large dynamic range and a low input coil inductance. These observations led to a SQUID design with low SQUID inductance, high critical current density, and large junction capacitance. A first successful production run resulted in SQUIDs with a flux noise of $1.210^{-7} \Phi_{0} / \mathrm{Hz}^{1 / 2}$ at $4.2 \mathrm{~K}$. It is expected that operation at $100 \mathrm{mK}$ will reduce this this noise level by about a factor 3. The SQUIDs are equipped with an output transformer to match the SQUID to a $50 \Omega$ cable. The input coil inductance of these SQUIDs equals $0.7 \mathrm{nH}$ for a current noise of $3.6 \mathrm{pA} / \mathrm{Hz}^{1 / 2} @ 4.2 \mathrm{~K}$. This inductance is low enough to make cross-talk caused by common inductance negligible.

A rather careful and detailed analysis of the FDM system, taking into account limitations due to finite FLL gain-bandwidth and mixing due to SQUID alinearity, indicates that the FDM system allows for approximately 16 - 32 channels with the XEUS specifications to be multiplexed into one SQUID channel. Making use of the TDM system prognoses made at NIST ${ }^{25}$ we arrive at an estimate of $3.5-7$ channels for TDM with XEUS type specifications. So FDM does have a distinct advantage over TDM for this type of application.

\section{ACKNOWLEDGEMENTS}

Part of this work is supported by a research grant from the Dutch Organization for Scientific Research (NWO), and by contract 15850/01/NL/HB of ESA's technological research program.

\section{REFERENCES}

1. S.H. Moseley, J.C. Mather, and D.McCammon, Thermal detectors as X-ray spectrometers, J. Appl. Phys. 56 (1984) 1257.

2. C.K. Stahle, C.A. Allen, K.R. Boyce, R.P. Brekosky, G.V. Brown, J. Cottam, E. Figuera-Feliciano, M. Galeazzi, J.D. Gygax, M.B. Jacobson, R.L. Kelley, D. Liu, D. McCammon, R.A. McClanahan, S.H. Moseley, F.S. Porter, L.E. Rocks, A.E. Szymkowiak, and J.E. Vaillancourt, The next generation microcalorimeter array of XRS on Astro-E2, Nucl. Instr. and Meth. A 520, 466, 2004.

3. K.D. Irwin, An application of electrothermal feedback for high resolution resolution cryogenic particle detection, Appl. Phys. Lett. 66, 1998, 1995.

4. K.D. Irwin, G.C. Hilton, D.A. Wollman, and J.M. Martinis, Thermal-response time of superconducting transition-edge microcalorimeters, J. Appl. Phys. 83, 3978 (1998)

5. M.A. Lindeman, R.P. Brekosky, E. Figueroa-Feliciano, F.M. Finkbeiner, M. Li, C.K. Stahle, C.M. Stahle, and N. Tralshawala, Performance of Mo/Au TES Microcalorimeters, AIP.Conf.Proc 605, 203, 2002, Ed. F.S. Porter, M. Galeazzi, and C.K. Stahle.

6. W.M. Bergmann Tiest, H.F.C. Hoevers, W.A. Mels, M.L. Ridder, M.P. Bruijn, P.A.J. de Korte, and M.E. Huber, 
Performance of X-ray microcalorimeters with an energy resolution below $4.5 \mathrm{eV}$ and $100 \mu$ s response time, AIP.Conf.Proc 605, 199, 2002, Ed. F.S. Porter, M. Galeazzi, and C.K. Stahle.

7. H.F.C. Hoevers, A.C. Bento, M.P. Bruijn, L. Gottardi, M.A.N. Korevaar, W.A. Mels, and P.A.J. de Korte,

Thermal fluctuation noise in a voltage biased superconducting transition edge thermometer, Appl. Phys. Lett. 77, 4422,2000

8. W.B. Bergmann Tiest, M.P. Bruijn, H.F.C. Hoevers, P.A.J. de Korte, J. van der Kuur, W.A. Mels, Understanding TES microcalorimeter noise and energy resolution, Nucl. Instr. and Meth. A 520, 329, 2004.

9. D.J. Fixen, S.H. Moseley, B. Cabrera, E. Figueroa-Feliciano, Pulse estimation in nonlinear detectors with non- stationary noise, Nucl. Instr. and Meth. A 520, 555, 2004.

10. M.P. Bruijn, N.H.R. Baars, W.M. Bergmann Tiest, A. Germeau, H.F.C. Hoevers, P.A.J. de Korte, W.A. Mels, M.L. Ridder, E. Krouwer, J.J. van Baar, and R.J. Wiegerink, Development of an array of transition edge sensors for application in X-ray astronomy, Nucl. Instr. and Meth. A 520, 443, 2004.

11. M.L. Ridder, M.P. Bruijn, H.F.C. Hoevers, A. Germeau, N.H.R. Baars, E. Krouwer, J.J. van Baar, and R.J. Wiegerink, Thermal design issues and performance of microcalorimeter arrays at sub-Kelvin temperatures, Proceedings of IEEE International Conference on Sensors 2003, 353, 2003

12. J.A. Chervenak, K.D. Irwin, E.N. Grossman, J.M. Martinis, C.D. Reintsema, and M.E. Huber, Superconducting multiplexer for arrays of transition edge sensors, Appl. Phys. Lett. 74 (1999), no. 26, 4043.

13. K.D. Irwin, M.D. Audley, J.A. Beall, J. Beyer, S. Deiker, W. Doriese, W. Duncan, G.C. Hilton, W. Holland, C.D. Reintsema, J.N. Ullom, L.R. Vale, and Y. Xu, In-focal-plane SQUID multiplexer, Nucl. Instr. and Meth. A 520, 544, 2004.

14. M. Kiviranta, H. Seppae, J. van der Kuur, and P. de Korte, Squid-based read-out schemes for microcalorimeter arrays, AIP.Conf.Proc 605, 295, 2002, Ed. F.S. Porter, M. Galeazzi, and C.K. Stahle.

15. J. van der Kuur, P.A.J. de Korte, P. de Groene, N.H.R. Baars, M.P. Lubbers, and M. Kiviranta, Implementation of frequency domain multiplexing in imaging arrays of microcalorimeters, Nucl. Instr. and Meth. A 520, 551, 2004

16. K.D. Irwin, SQUID multiplexers for transition-edge sensors, Physica C 368, 203, 2002

17. J. Yoon, J. Clarke, J. Gildemeister, A. Lee, M. Myers, P. Richards, and J. Skidmore, Single superconducting quantum interference device multiplexer for arrays of low-temperature sensors, Appl. Phys. Lett., 78, 371, 2001

18. M. Mitsuda, R. Fujimoto, M. Maegami, Y. Aruga, T. Oshima, S. Nakayama, S. Shoji, H. Kudo, Y. Yokoyama, T. Mihara, and H.M. Shimuzu, Multi-pixel read-out of transition-edge sensors using a multi-input SQUID, Nucl. Instr. and Meth. A 436, 252, 1999.

19. M.F. Cunningham, J.N. Ullom, T. Miyazaki, S.E. Labov, J. Clarke, T.M. Lanting, A.T. Lee, P.L. Richards, J. Yoon, and H. Spieler, High-resolution operation of frequency-multiplexed transisiton-edge photon sensors, Appl. Phys. Lett.

81, 159, 2002

20. J. van der Kuur, P.A.J. de Korte, H.F.C. Hoevers, M. Kiviranta, and H. Seppae, Performance of an x-ray microcalorimeter under AC biasing, Appl. Phys. Lett. 81, 4467, 2002

21. N.H.R. Baars, J. van der Kuur, M.P. Lubbers, and P.A.J. de Korte, First results of a TES microcalorimeter AC-biased at $500 \mathrm{kHz}$, Nucl. Instr. and Meth. A 520, 574, 2004

22. T.M. Lanting, private communication, 2003

23. A.T. Lee, private communication, 2003.

24. M. Kiviranta, J. Penttilae, L. Groenberg, J. Hassel, A. Virtanen, and H. Seppae, Design and performance of multiloop and washer SQUIDs intended for sub-Kelvin operation, Supercond. Sci. Technol., 17, 285, 2004.

25. J.N. Ullom, J.A. Beall, A. Clark, S. Deiker, W. Doriese, W. Duncan, L. Ferriera, G.C. Hilton, K. Irwin, N. Miller, C. Reintsema, L.R. Vale, and Y. Xu, TES array and read-out development at NIST, $2^{\text {nd }}$ International Workshop on Transition Edge Sensor Device Physics, March 18 - 19, 2004, Miami, Florida, http://origins.colorado.edu/ deiker/tes/index.cgi 